\title{
The effect of crop parameters on mechanical properties of oil palm fruitlets
}

\begin{abstract}
Oil palm fruitlets are highly susceptible to mechanical damage during harvesting and handling. In this study, the effect of crop parameters and two loading orientations on mechanical properties of oil palm fruitlets were investigated. The investigated crop parameters were four mass categories (11-11.9, 12-12.9, 13-13.9 and 14-14.9 g), two varieties (Tenera and Dura) and three ripeness stages (underripe, ripe and overripe). For impact loading, both vertical and horizontal loading orientations were studied. The investigated mechanical properties were rupture force, rupture energy, deformation at rupture and specific deformation. The mechanical properties were measured using a pendulum impact load test device. A total of 540 fruitlet samples extracted from 18 fresh fruit bunches (FFB) were employed. The results showed that the values of rupture force and energy for all mass categories under the vertical loading orientation were lower than their values under the horizontal loading orientation. The maximum rupture forces of 697.1 and $939.4 \mathrm{~N}$ were recorded for the highest mass category (14-14.9 g) under the vertical and horizontal loading orientations, respectively. The results also showed that the values of all mechanical properties belong to Tenera variety were higher than that of Dura variety under the both loading orientations. In terms of the ripeness stage, the rupture force and energy of oil palm fruitlets decreased as the ripeness stage increased. The maximum rupture force and energy of $940 \mathrm{~N}$ and $2.95 \mathrm{Nm}$ were recorded for ripe stage under the horizontal loading orientation, respectively. The results from this study would be very useful in designing a proper harvesting and in-field transportation systems to minimize crop damage due to impact loading.
\end{abstract}

Keyword: Mechanical properties; Impact loading; Oil palm fruitlets; Rupture force; Palm oil 